\title{
MUC2 Is a Molecular Marker for Pseudomyxoma Peritonei
}

Jerome T. O'Connell, M.D., Cammy M. Hacker, B.A., Sanford H. Barsky, M.D.

Department of Pathology, UCLA School of Medicine, Los Angeles, California

Pseudomyxoma peritonei, a syndrome first described by Rokitansky in 1842, is an enigmatic, often fatal intra-abdominal disease characterized by dissecting gelatinous ascites and multifocal peritoneal epithelial implants secreting copious globules of extracellular mucin. Although much past interest in the syndrome has focused on the question of whether the disease arises from primary appendiceal or ovarian mucinous tumors of varying malignant potential, the accumulation of extracellular mucin with its resulting obstruction of abdominal viscera and adhesion formation is one major cause of this disease's morbidity and mortality irrespective of the origin or transformed status of the epithelium secreting it. Because of this and because of the recent discovery and cloning of a series of specific mucin genes responsible for mucin secretion and extracellular deposition, we decided to analyze cases of pseudomyxoma peritonei with specific mucin cDNAs and corresponding antibodies to identify a characteristic marker for this disease which ultimately might be targeted therapeutically. Our study specifically investigated MUC2 and MUC5AC because these two mucins possessed the physicochemical property of being gel-forming, a property exhibited by pseudomyxoma peritonei grossly. Expression of MUC2 and MUC5AC in pseudomyxoma peritonei and in accompanying and nonaccompanying appendiceal and ovarian mucinous neoplasms were analyzed by in situ hybridization, immunocytochemistry and digital image analysis. A striking overexpression of both MUC2 and MUC5AC was observed in nearly all cases of pseudomyxoma peritonei of unknown and appendiceal origin. In these cases, however, MUC2 gene expression was more prominent. The mucin:cell ratio averaged 10 to 1 in these cases. The primary ovarian mucinous

Copyright (C) 2002 by The United States and Canadian Academy of Pathology, Inc.

VOL. 15, NO. 9, P. 958, 2002 Printed in the U.S.A.

Date of acceptance: May 25, 2002.

Address reprint requests to: Sanford H. Barsky, M.D., Department of Pathology, UCLA School of Medicine, Los Angeles, CA 90024; e-mail: sbarsky@ucla.edu; Fax: 310-441-1248.

DOI: $10.1097 / 01 . M P .0000026617 .52466 .9 F$ tumors, some of which exhibited pseudomyxoma ovarii and/or peritoneal implants but not classic pseudomyxoma peritonei, in contrast, expressed only MUC5AC and gave rise to implants where the mucin:cell ratio averaged only 1 to 1 . MUC2 overexpression then supported an intestinal rather than ovarian origin for true pseudomyxoma peritonei, irrespective of whether an appendiceal primary was documented. In all cases studied, the fidelity of MUC2 and MUC5AC expression held irrespective of the degree of malignant transformation which was present. MUC2 is therefore a reliable molecular marker for pseudomyxoma peritonei.

KEY WORDS: Appendiceal mucinous tumors, Disseminated peritoneal adenomucinosis, Gelatinous ascites, MUC2, MUC5AC, Mucin, Ovarian mucinous tumors, Peritoneal mucinous carcinomatosis, Pseudomyxoma peritonei.

Mod Pathol 2002;15(9):958-972

Pseudomyxoma peritonei, a syndrome first described by Rokitansky in 1842 (1), is an enigmatic, often fatal intra-abdominal disease characterized by dissecting mucinous ascites and multifocal peritoneal epithelial implants secreting copious globules of extracellular mucin (2-5). In classic cases over $90 \%$ of the globular masses are mucin by volume. The syndrome in the past was considered to arise either from primary appendiceal or ovarian mucinous tumors (6-10) but recent evidence has favored an appendiceal origin (11-19). It has been recently demonstrated that "traditional pseudomyxoma peritonei" includes three pathologically, surgically, and prognostically distinctive types of tumors with varying degrees of malignant transformation. These include disseminated peritoneal adenomucinosis, peritoneal mucinous carcinomatosis and peritoneal mucinous carcinomatosis with intermediate or discordant features $(15,20)$ so the degree of transformation of the mucinous epithelium is important in determining the behavior of the disease process. Disseminated peritoneal adenomucinosis has a more favorable response to cytoreductive surgery and intraperitoneal chemo- 
therapy (20). Mucin accumulation is the overwhelming problem in disseminated peritoneal adenomucinosis whereas widespread dissemination and infiltration of carcinoma in addition to mucin accumulation is the problem in peritoneal mucinous carcinomatosis. Because of this and because of the recent discovery and cloning of a series of specific mucin genes responsible for mucin secretion and extracellular deposition $(21,22)$, we decided to analyze cases of pseudomyxoma peritonei with specific mucin cDNAs and corresponding antibodies to investigate whether specific mucin expression could provide a molecular marker for this disease. Our study specifically investigated MUC2 and MUC5AC because these two mucins possessed the physicochemical property of being gel-forming $(23,24)$, a property exhibited by pseudomyxoma peritonei grossly. Our study also focused on MUC2 and MUC5AC because recent studies had observed differential expression of these 2 mucins in gastrointestinal $v$ ovarian neoplasms (25).

\section{MATERIALS AND METHODS}

\section{Selection of Cases}

We retrospectively retrieved 100 cases of pseudomyxoma peritonei, 60 of which had a past or synchronous mucinous tumor of the appendix and 40 of which had no such documentation because either the appendix was not sampled or represented pseudomyxomatous recurrences without clinical information of the initial surgery/Of the 40 cases with no documented appendiceal primary, 10 exhibited ovarian involvement. In the vast majority of these 10 cases morphological analysis was equivocal and we could not determine by routine light microscopy whether we were dealing with an ovarian primary or an ovarian metastasis. Of the 60 cases with a mucinous tumor of the appendix, 24 had synchronous ovarian involvement. We also retrospectively retrieved 15 cases of solitary mucinous tumors of the appendix without pseudomyxoma peritonei and 75 cases of primary ovarian mucinous tumors, 15 of which had peritoneal implants and 5 of which had pseudomyxoma ovarii (extracellular mucin pools within ovarian stroma) but not generalized pseudomyxoma peritonei. The primary ovarian mucinous tumors and the mucinous tumors of the appendix retrieved for study all included benign, borderline and malignant histologies. For the cases of pseudomyxoma peritonei, we applied a recent correlative morphologic and prognostic classification scheme $(15,20)$. Specifically cases were classified into either the disseminated peritoneal adenomucinosis, peritoneal mucinous carcinomatosis or peritoneal mucinous carcinomatosis with intermediate or discordant features categories.
Pseudomyxoma peritonei cases categorized as disseminated peritoneal adenomucinosis demonstrated abundant mucin containing scant strips of mucinous epithelium with mild cytologic atypia and rare mitoses. The glandular epithelium was simple and nonstratified to focally proliferative. Cases categorized as peritoneal mucinous carcinomatosis had more abundant epithelium demonstrating marked cytologic atypia. This epithelium formed malignant glands or nest or occurred as individual malignant cells including signet ring cells. Cases categorized as peritoneal mucinous carcinomatosis with intermediate or discordant features included cases of an intermediate category that demonstrated features of both disseminated peritoneal adenomucinosis and peritoneal mucinous carcinomatosis as well as cases showing discordant features. The histologic distribution of the selected cases are summarized in Table 1. Sections of normal appendix, normal peritoneum and normal ovary served as controls.

\section{Routine Microscopic and Histochemical Studies}

All cases were reviewed by us and the diagnoses recorded. Alcian blue staining $(\mathrm{pH}=2.5)$ which was hyaluronidase resistant was used to better visualize the extracellular mucin pools. This histochemical stain helped distinguish extracellular mucin accumulations from other non-mucinous extracellular proteinaceous accumulations. All of the retrieved cases of pseudomyxoma peritonei exhibited varying degrees of extracellular mucin accumulation by Alcian blue staining.

\section{Immunocytochemical Studies}

The identity of the specific mucin(s) involved in each of the cases was investigated with antibodies to MUC2 and MUC5AC. Anti-MUC2 (Research Diagnostics, Inc., Flanders, NJ), a murine monoclonal 0 1 20

TABLE 1. Histologic Distribution of Studied Cases

\begin{tabular}{lc}
\hline \multicolumn{1}{c}{ Category } & \# Cases \\
\hline Pseudomyxoma peritonei & 100 \\
Disseminated peritoneal adenomucinosis & 70 \\
Peritoneal mucinous carcinomatosis with & 20 \\
intermediate or discordant features & \\
Peritoneal mucinous carcinomatosis & 10 \\
Associated with ovarian involvement & 34 \\
Associated with mucinous tumor of the appendix & 60 \\
Solitary mucinous tumors of the appendix & 15 \\
Benign & 3 \\
Borderline & 8 \\
Malignant & 4 \\
Primary ovarian mucinous tumors & 75 \\
Benign & 35 \\
Borderline & 25 \\
Malignant & 15 \\
Associated with peritoneal implants & 15 \\
Associated with pseudomyxoma ovarii & 5 \\
\hline
\end{tabular}


antibody (clone Ccp58) made to a synthetic peptide corresponding to a site on the MUC2 human core protein, and which was non-reactive to both MUC1 and MUC5AC, was used at a dilution of 1/100-1/ 200. Anti-MUC5AC (Research Diagnostics, Inc.), a murine monoclonal antibody (clone 45M1) made to a peptide core corresponding to a site on the MUC5AC human core protein, and which was nonreactive to both MUC1 and MUC2, was used at a dilution of $1 / 100-1 / 200$. Paraffin-embedded sections were deparaffinized, rehydrated and processed for antigen retrieval by citrate pretreatment ( $0.1 \mathrm{~mol} / \mathrm{L}$ sodium citrate buffer, $\mathrm{pH}$ 6.0) followed by microwave heating (26) for 10 minutes. Sheep anti-mouse IgG was used as a secondary antibody (1/200 dilution). Antigen-binding sites were then revealed by incubating with peroxidase polymerizing diaminobenzidine (3,3'-diaminobenzidine) producing insoluble brownish-black staining at sites of antigen presence. Immunoreactivity staining patterns were categorized as negative (absent staining), weak (positive in less than $5 \%$ of the cells) or strong (positive in more than $75 \%$ of the cells). All of the staining patterns fit into one of these three categories. We chose these categories after we had screened a number of cases and observed the pattern of staining. The category designated as weak (positive in less than $5 \%$ of the cells) was considered positive and not negative because the focal staining which was observed in less than $5 \%$ of the cells was strong in the cells themselves but the number of cells with positive signals was small. These positive cells were probably the scattered goblet cells present in some of the lesions. LLIf

\section{In Situ Hybridization Studies}

In situ hybridization studies (27) were conducted with riboprobes of MUC2 and MUC5AC. The pBluescript SK plasmid (Stratagene, La Jolla, CA) containing an EcoRI $90 \mathrm{bp}$ repeat region of the human MUC2 gene, termed HAM1 (28) was linearized with $X b a \mathrm{I}$ for antisense strand preparation from the T7 promotor and with HindIII for sense strand preparation from the T3 promotor. A pCRII plasmid (Invitrogen, Carlsbad, CA) containing an EcoRI $298 \mathrm{bp}$ tandem repeat fragment of the 3' end of the MUC5AC gene, was processed for riboprobe preparation identically except that the SP6 promotor was used for sense probe preparation. $\left[{ }^{35} \mathrm{~S}\right] \mathrm{UTP}-$ labeled RNA transcripts were synthesized at concentrations of $4-6 \times 10^{5} \mathrm{cpm} / \mu \mathrm{l}$. Paraffinembedded sections were deparaffinized, rehydrated, washed in $0.5 \times$ SSC and digested with proteinase $\mathrm{K}$ at room temperature for 10 minutes. The sections were acetylated using $0.25 \%$ acetic anhydride and $0.1 \mathrm{~m}$ triethanolamine $\mathrm{HCl}$, rinsed with $0.5 \times$ SSC and dehydrated with ethanol and
TABLE 2. Expression of MUC2/MUC5AC in Different Tumors

\begin{tabular}{lcc}
\hline \multirow{2}{*}{ Category } & \multicolumn{2}{c}{ AB (IS) } \\
\cline { 2 - 3 } & MUC2 & MUC5AC \\
\hline Pseudomyxoma peritonei & $98(95)$ & $95(90)$ \\
Disseminated peritoneal adenomucinosis & $100(98)$ & $98(96)$ \\
Peritoneal mucinous carcinomatosis with & $95(90)$ & $90(80)$ \\
$\quad$ intermediate or discordant features & & \\
Peritoneal mucinous carcinomatosis & $90(80)$ & $80(70)$ \\
Associated with ovarian involvement & $97(97)$ & $94(94)$ \\
Associated with mucinous tumor of the & $97(97)$ & $93(93)$ \\
appendix & & \\
Solitary mucinous tumors of the appendix & $100(100)$ & $100(100)$ \\
Benign & $100(100)$ & $100(100)$ \\
Borderline & $100(100)$ & $100(100)$ \\
Malignant & $100(100)$ & $100(100)$ \\
Primary ovarian mucinous tumors & $16(16)$ & $100(96)$ \\
Benign & $9(9)$ & $100(97)$ \\
Borderline & $16(16)$ & $100(96)$ \\
Malignant & $20(20)$ & $100(93)$ \\
Associated with peritoneal implants & $20(20)$ & $100(100)$ \\
Associated with pseudomyxoma ovarii & $0(0)$ & $100(100)$ \\
\hline
\end{tabular}

Results expressed as percentage of cases exhibiting any degree of positivity including weak or focal positivity. AB represents immuno results and IS represents in situ hybridization results, the latter contained within parentheses, for MUC2 and MUC5AC.

finally air dried. A MUC riboprobe was applied in a hybridization mixture containing deionized formamide $(50 \%)$, dextran sulfate $(10 \%)$, tRNA $(0.5 \mathrm{mg} /$ $\mathrm{mL})$, salmon sperm DNA ( $1 \mathrm{mg} / \mathrm{mL}), 10 \mathrm{~mm}$ dithiothreitol (DTT), $0.3 \mathrm{~m} \mathrm{NaCl}, 5 \mathrm{~mm}$ EDTA, $20 \mathrm{~mm}$ Tris- $\mathrm{HCl}$ and $10 \mathrm{~mm} \mathrm{NaPO}_{4}(\mathrm{pH}$ 6.8). The mixture was heated to $65^{\circ} \mathrm{C}$ for $15 \mathrm{~min}$ and chilled on ice. Fresh DTT was added to achieve a concentration of $20 \mathrm{~mm}$. The 120 of the mixture was applied to each section and parafilm cover slips were applied. Hybridizations were carried out in humidified chambers overnight at $55^{\circ} \mathrm{C}$. Coverslips were removed in $5 \times \mathrm{SSC}, 10 \mathrm{~mm}$ DTT at $55^{\circ} \mathrm{C}$. Sections were washed three times in $2 \times$ SSC containing 2-mercaptoethanol and EDTA, treated with RNase A, and washed in $0.1 \times \mathrm{SSC}$ at $62^{\circ} \mathrm{C}$ for 3 hours (high stringency). Slides were then washed for $5 \mathrm{~min}$ at room -temperature in $0.5 \times$ SSC without 2-mercaptoethanol or EDTA. The sections were dehydrated with ethanol and air dried. The slides were exposed to Ilford K5D emulsion and stored in the dark at $4^{\circ} \mathrm{C}$ until developed which took at least 10-14 days.

\section{Digital Image Analysis and Visual Estimate Studies}

Digital image analysis was used to quantitate the amount of extracellular mucin present and the ratio of extracellular mucin to cells. In the in situ hybridization studies, digital image analysis was used to compare the signal intensities of the antisense probe with the signal intensities of the sense probe (background) over the corresponding areas of neoplastic and control epithelium. Signal intensities 

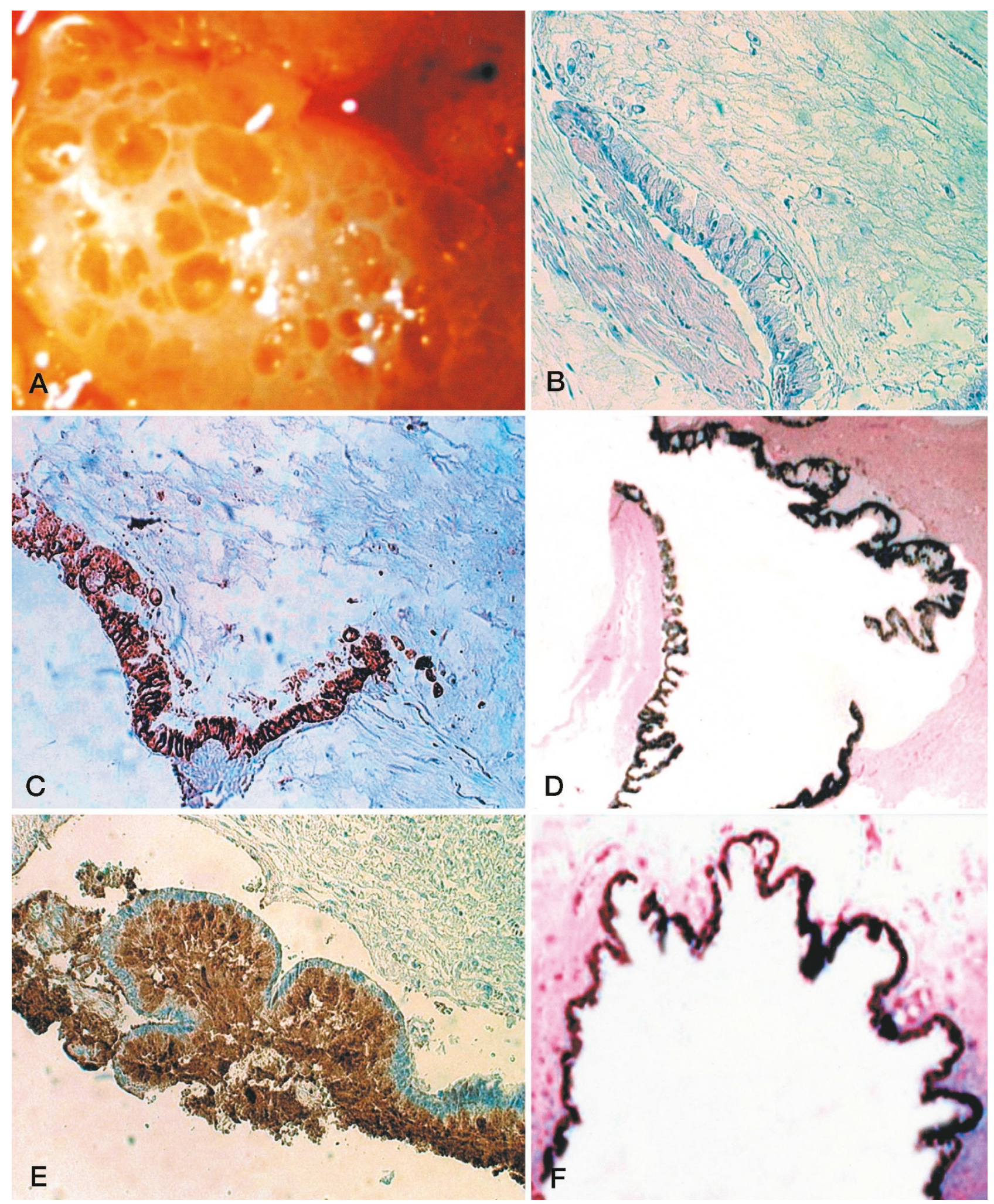

E

(3)

FIGURE 1. The features of pseudomyxoma are depicted from a representative case. The typical gross appearance of pseudomyxoma peritonei illustrates the so-called "jelly-belly" (A); the hallmark of the disease is copious accumulations of mucin deposits in which the intestinal lining cells are sometimes difficult to see (B); anti-MUC2 however clearly delineates the intestinal lining cells responsible for this massive mucin secretion and accumulation. The extracellular mucin deposits paradoxically are not immunoreactive because their extensive glycosylation blocks antibody access to the core protein (C); antisense MUC2 in situ hybridization demonstrates intense MUC2 signals within this lining epithelium (D); similarly antiMUC5AC also delineates the intestinal lining cells responsible for mucin secretion; the extracellular mucin deposits close to the cells are, however, immunoreactive presumably because their glycosylation status is less restrictive to antibody access than was MUC2 (E); antisense MUC5AC in situ hybridization demonstrates less intense but still significant MUC5AC signals within this lining epithelium (F). A, gross photograph; B, hematoxylin and eosin, x250; C, anti-MUC2, immunoperoxidase, x250; D, MUC2 antisense riboprobe, x250; E, anti-MUC5AC, immunoperoxidase, x450; F, MUC5AC antisense riboprobe, $\mathrm{x} 250$ 
were expressed as antisense fold increase over sense. Signal intensity per cell was calculated. Digital image analysis utilized a digital imaging system, composed of a Leitz Dialux microscope linked to a Vidicon camera, an IBM PC with PCVision digitizer, and Microscience software. In addition to digital image analysis, we estimated the mucin/cell ratio by visually scanning fields under the light microscope and making a semiquantitative estimate of the ratio in the different mucinous lesions.

\section{Statistical Analysis}

Experiments were performed by counting $10 \mathrm{mi}-$ croscopic fields per case with the appropriate magnification (mucin:cell ratio, x250; in situ hybridization, x250 and x450) and results were expressed as mean \pm standard deviation. Other experiments combined the results of the individual cases into the appropriate groups for analysis. All results were analyzed with standard tests of significance, including the 2-tailed Student's $t$ test and a one-way analysis of variance (ANOVA).

\section{Institutional Certifications}

Since this was a retrospective study, informed patient consent was waived. Approval for this study was obtained from the UCLA Human Subject Protection Committee and the UCLA Institutional Biosafety Committee.

\section{RESULTS}

\section{Overview}

A striking overexpression of both MUC2 and MUC5AC was observed in nearly all cases of pseudomyxoma peritonei of unknown and appendiceal origin (Fig. 1A-F) $(P<.01)$. In these cases, however, MUC2 gene expression, by in situ hybridization, was uniformly more prominent (Fig. 1D) than MUC5AC expression (Fig. 1F) $(P<.05)$. In analyzing the associated mucinous tumors of the appendix, a similar overexpression of both MUC2 and MUC5AC was observed $(P<.01)$.

The solitary mucinous tumors of the appendix without pseudomyxoma peritonei similarly exhibited a striking overexpression of both MUC2 and MUC5AC in all cases (Fig. 2A, C, E) $(P<.01)$. Primary ovarian mucinous tumors including benign (Fig. 2B), borderline and malignant ovarian tumors essentially did not express MUC2 (Fig. 2D) but overexpressed MUC5AC (Fig. 2F) $(P<.01)$.

Nearly all cases of pseudomyxoma peritonei (Fig. $3 \mathrm{~A})$ that metastasized to the ovary gave a pattern of mucin gene expression (Fig. 3C, E) identical to that exhibited by mucinous tumors of the appendix. Primary malignant ovarian mucinous tumors (Fig.
3B), on the other hand, largely did not express MUC2 (Fig. 3D) but only MUC5AC (Fig. 3F) even when they were associated with peritoneal implants $(P<.01)$.

Nearly all cases of pseudomyxoma peritonei overexpressed MUC2 which therefore supported an intestinal rather than ovarian origin for true pseudomyxoma peritonei, irrespective of whether an appendiceal primary was documented. When morphological analysis was equivocal, both MUC2 immunostaining and MUC2 in situ hybridization were useful in distinguishing an intestinal $v$ ovarian primary in problematic cases involving the ovary.

In the cases expressing MUC2, the fidelity and constitutiveness of MUC2 expression held irrespective of the degree of malignant transformation which was present. This was illustrated in the normal appendix (Fig. 4A) where only the goblet cells exhibited MUC2 immunoreactivity (Fig. 4B); in pseudomyxoma peritonei of benign appearing lining cells (Fig. 4C) where MUC2 expression was present (Fig. 4D); in borderline mucinous tumors of the appendix (Fig. 4E) where MUC2 was strongly immunoreactive (Fig. 4F); and frank adenocarcinomas of the appendix as this case of signet ring carcinoma (Fig. 4G) which also exhibited strong MUC2 immunoreactivity (Fig. 4H). Interestingly the surface cells of the ovary and the mesothelial cells of the peritoneum did not express MUC2 (data not shown).

In situ hybridization studies revealed 10 fold antisense MUC2 signals (Fig. 5A) over sense (Fig. 5B) present in all of the transformed lining intestinal cells of pseudomyxoma peritonei. The normal appendix similarly showed 10 fold antisense MUC2 signals but only in its goblet cells (Fig. 5C-D).

- The mucin:cell ratio averaged 10 to 1 in all of the cases of pseudomyxoma peritonei which were studied whether there was an associated mucinous tumor of the appendix or ovarian involvement (Fig. 6A) $(P<.01)$. Ovarian neoplasms, in contrast, gave rise to implants where the mucin:cell ratio was only 1 to 1 (Fig. $6 \mathrm{~A})(P<.05)$.

In nearly all of the cases of pseudomyxoma peritonei, whether there was an associated mucinous tumor of the appendix or ovarian involvement (Fig. 6B), in situ hybridization studies with antisense MUC2 and MUC5AC revealed strong MUC2 and MUC5AC signals (10 fold and 4-5 fold increases respectively) $(P<.01 ; P<.05)$. Ovarian neoplasms, in contrast, gave rise to implants where only the antisense MUC5AC revealed strong MUC5AC signals. In these cases MUC5AC signals were slightly stronger than in the cases of pseudomyxoma peritonei but this difference was not statistically significant (Fig. $6 \mathrm{~B})(P>0.1)$. In 

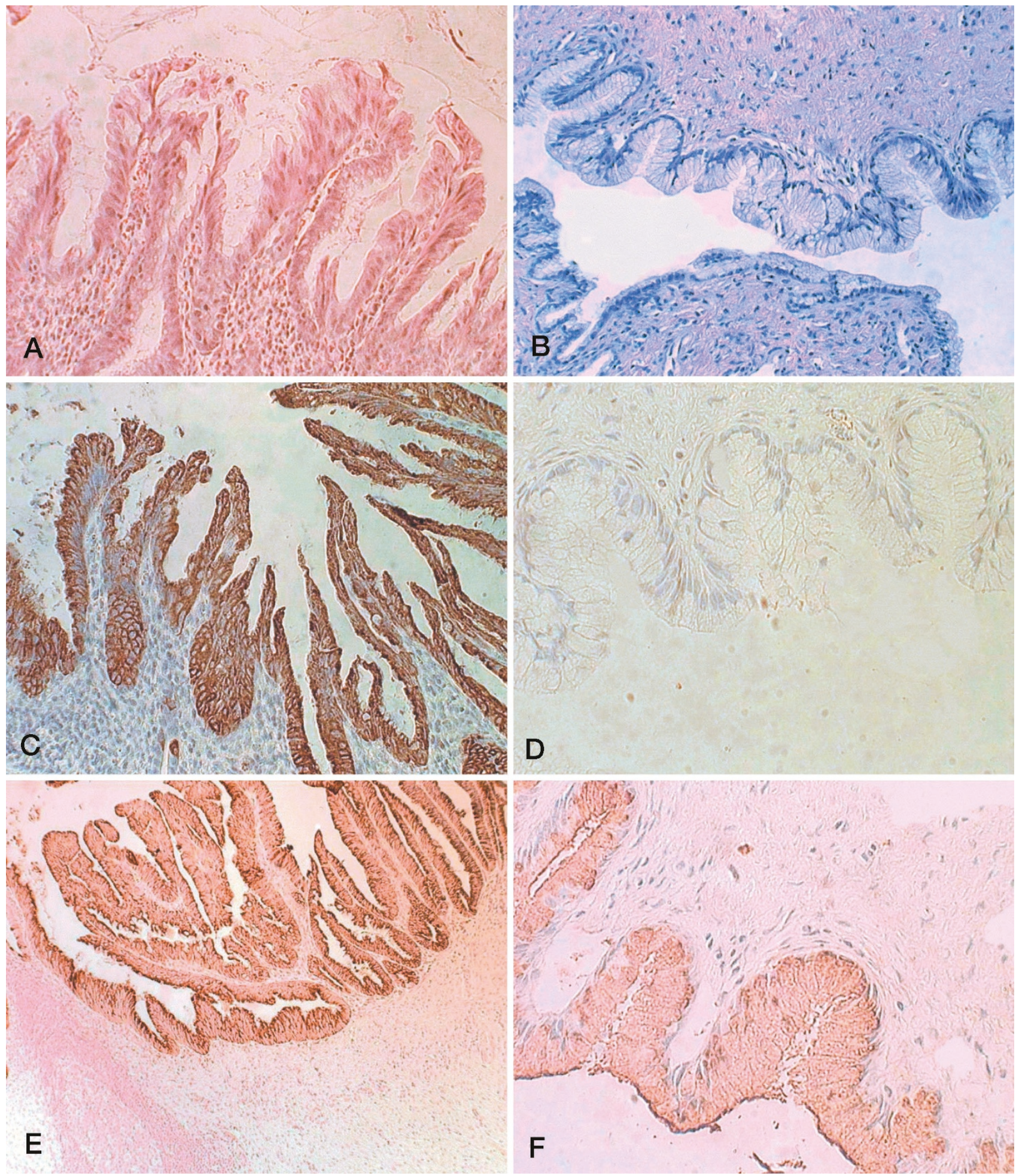

E

FIGURE 2. The mucin immunocytochemical profile of a solitary mucinous tumor of the appendix, in this case, a mucinous borderline tumor (A), contrasts with the mucin immunocytochemical profile of a primary ovarian mucinous tumor, in this case a mucinous cystadenoma (B); intense MUC2 immunoreactivity is observed in the mucinous tumor of the appendix (C) just as it was observed in pseudomyxoma peritonei; the primary ovarian mucinous cystadenoma is MUC2 non-immunoreactive (D); both tumors exhibit MUC5AC immunoreactivity (E,F). A, hematoxylin and eosin, x250; B, hematoxylin and eosin, x250; C, anti-MUC2, immunoperoxidase, x250; D, anti-MUC2, immunoperoxidase, x250; E, anti-MUC5AC, immunoperoxidase, x250; F, anti-MUC5AC, immunoperoxidase, x250

situ MUC2 and MUC5AC expressions were independent of the degrees of malignant transformation present within each category (Fig. 6C) $(P>$ $.1)$.

\section{Detailed Findings}

Our detailed results for all the mucinous tumors studied are summarized in Table 1 and Table 2 and are as follows: 

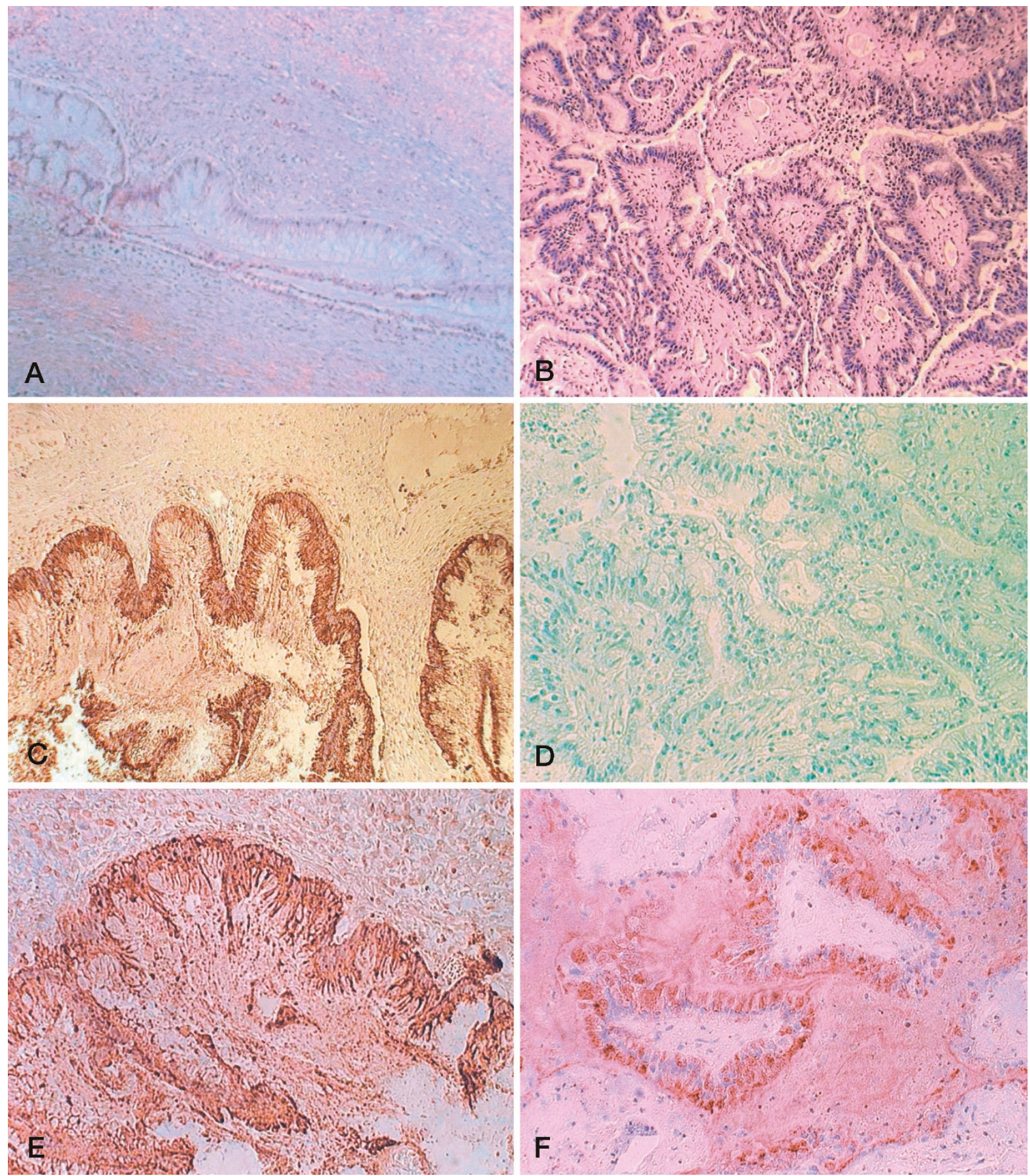

FIGURE 3. The mucin immunocytochemical profile of pseudomyxoma peritonei with metastatic involvement of the ovary (A) contrasts with that of a primary ovarian mucinous adenocarcinoma (B) exhibiting papillary areas in this section — other areas were invasive; intense MUC2 immunoreactivity is observed in the metastases to the ovary (C); the primary ovarian mucinous adenocarcinoma is MUC2 non-immunoreactive (D); both tumors again share MUC5AC immunoreactivity (E,F). A, hematoxylin and eosin, x250; B, hematoxylin and eosin, x250; C, anti-MUC2, immunoperoxidase, x250; D, anti-MUC2, immunoperoxidase, x250; E, anti-MUC5AC, immunoperoxidase, x250; F, anti-MUC5AC, immunoperoxidase, $\mathrm{x} 250$

\section{Pseudomyxoma Peritonei}

Cases of pseudomyxoma peritonei distributed among the disseminated peritoneal adenomucinosis, peritoneal mucinous carcinomatosis and peri- toneal mucinous carcinomatosis with intermediate or discordant features categories according to the published criteria $(15,20)$ but a clear majority of these cases fell within the disseminated peritoneal 

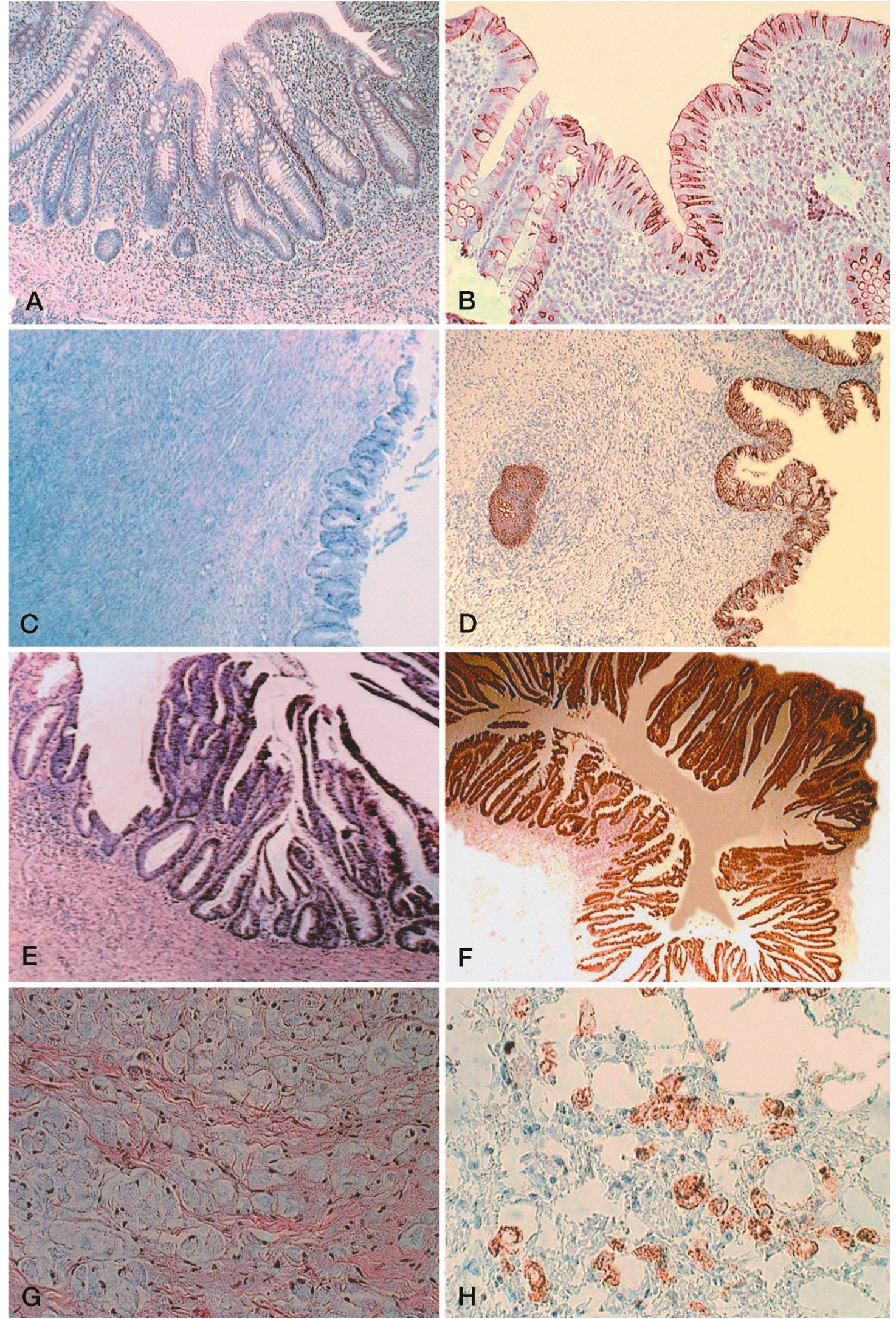

FIGURE 4. Different degrees of appendiceal malignant transformation all exhibit high constitutive expression of MUC2. Normal appendix (A) exhibits focal expression of MUC2 in goblet cells (B); a pseudomyxoma peritonei implant from an appendiceal adenoma (C) expresses high levels of MUC2 (D); a borderline mucinous tumor of the appendix (E) similarly exhibits high MUC2 (F); a poorly differentiated signet ring adenocarcinoma arising within the appendix (G) similarly expresses high MUC2 (H). A, hematoxylin and eosin, x250; B, anti-MUC2, immunoperoxidase, x250; C, hematoxylin and eosin, x250; D, anti-MUC2, immunoperoxidase, x250; E, hematoxylin and eosin, x250; F, anti-MUC2, immunoperoxidase, x250; G, hematoxylin and eosin, x250; H, anti-MUC2, immunoperoxidase, x250 


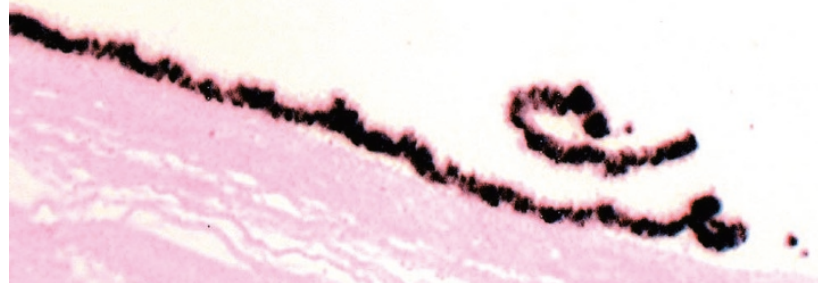

A

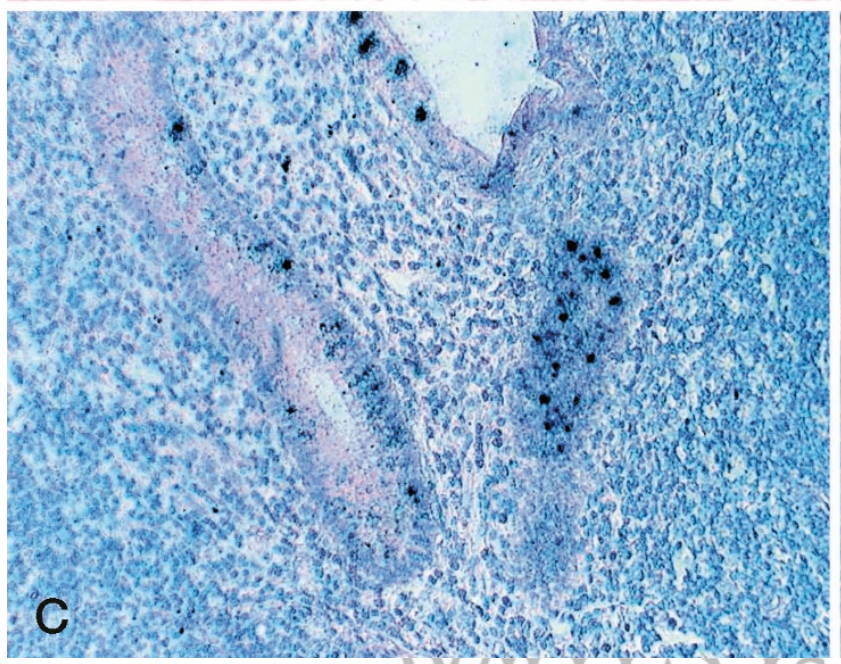

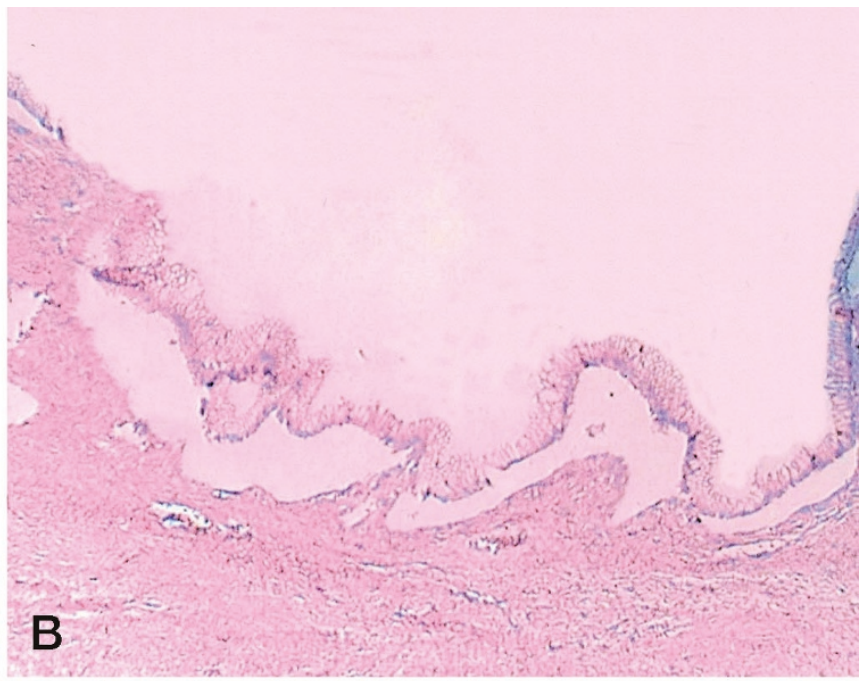

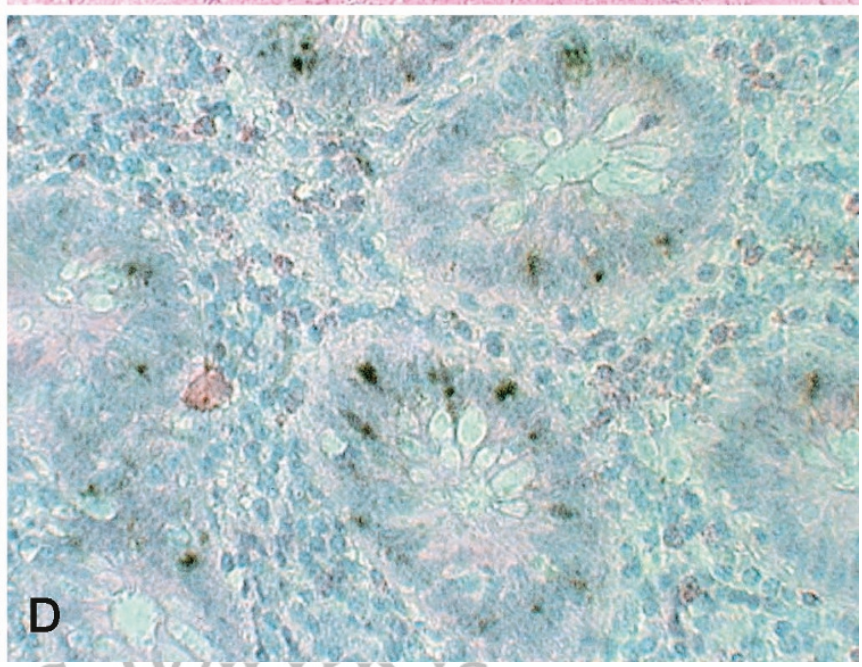

FIGURE 5. MUC2 in situ hybridization in pseudomyxoma peritonei compared to normal appendix. Antisense signals (A) are 10 fold over sense (B) in pseudomyxoma peritonei; similarly intense antisense signals are seen over goblet cells in normal appendix (C,D). A, MUC2 antisense riboprobe, x250; B, MUC2 sense riboprobe, x250; C, MUC2 antisense riboprobe, x250; MUC2 antisense riboprobe, x450

adenomucinosis category (Table 1) (Fig. 4C-D). Cases of pseudomyxoma peritonei regardless of histology were uniformly characterized by voluminous pools of extracellular mucin (Fig. 1A, Fig. 6A) containing variable amounts of predominately intestinal type lining cells (Fig. 1B) which involved the peritoneal surfaces. In 34/100 cases there was ovarian involvement (Fig. 3A, C, E) which was bilateral in 28 of these cases. In the 60 cases with a history of or synchronous mucinous tumor of the appendix, the cytologic features of the cells on the peritoneum and ovary were virtually identical to those seen in the mucinous tumor of the appendix. 98 of 100 cases of pseudomyxoma peritonei exhibited a diffuse and intense pattern of cytoplasmic MUC2 immunoreactivity (Fig. 1C). The extracellular mucin deposits adjacent to the immunopositive cells did not exhibit MUC2 immunoreactivity (Fig. 1C). In the 34 cases of pseudomyxoma peritonei with ovarian involvement (Fig. 3A, C, E) and in the 60 cases with an associated mucinous tumor of the appen- dix there were no differences in MUC2 immunoreactivity among the different sites. 95 of the 100 cases of pseudomyxoma peritonei exhibited MUC5AC immunoreactivity. The pattern of immunoreactivity was similar to that exhibited by MUC2 and predominantly cytoplasmic. There was however some extracellular mucin immunoreactivity adjacent to the intestinal type lining cells (Fig. 1E). In the 34 cases of pseudomyxoma peritonei with ovarian involvement and in the 60 cases with an associated mucinous tumor of the appendix there were no differences in MUC5AC immunoreactivity among the different sites. In 95 of the 100 cases of pseudomyxoma peritonei in situ hybridization studies revealed intense MUC2 signals (Fig. 1D; Fig. $5 \mathrm{~A}-\mathrm{B}) .90$ of these 100 cases also exhibited MUC5AC signals (Fig. 1F). In the 34 cases of pseudomyxoma peritonei with ovarian involvement and in the 60 cases with an associated mucinous tumor of the appendix there was $100 \%$ consistency among the in situ hybridization findings. The MUC2 signals were 
generally more intense than the MUC5AC signals (Fig. 1D, F, Fig. 6B). There was no difference in either the MUC2 or MUC5AC signals with the degrees of malignant transformation (Fig. 6C).

\section{Mucinous Tumors of the Appendix}

The solitary mucinous tumors of the appendix (Fig. 2A) consisted of 3 adenomas, 8 borderline tumors and 4 adenocarcinomas. It should be pointed out that the difference between adenoma and borderline tumor in the appendix was somewhat arbitrary and subjective. We mainly used the relative degree of nuclear atypia and degree of cellular stratification to categorize these lesions. The adenomas had benign appearing nuclei which were predominately basally oriented and were mainly single cell layered. The borderline tumors were characterized by either a villous (5 cases), tubular (1 case), or tubulovillous (2 cases) architecture. The borderline tumors were composed of 1-2 layers of hyperchromatic, elongated nuclei which were arranged perpendicularly to the lamina propria. Each case was associated with marked distension of the appendiceal wall and extravasation of mucin containing cytologically identical cells on the serosal surface. The adenocarcinomas, on the other hand, were associated with severe nuclear anaplasia and the presence of invasion of the appendiceal wall by malignant cells that was associated with a desmoplastic reaction. These cases were also associated with the presence of pools of mucin/containing cytologically identical malignant cells on the serosa of the appendix. In 1 of the 4 malignant cases, the histology was that of a signet ring carcinoma (Fig. $4 \mathrm{G}, \mathrm{H})$. It is important to emphasize that independent of any subjective classification scheme, all of the primary mucinous tumors of the appendix overexpressed MUC2. All of the 15 solitary mucinous tumors of the appendix (Fig. 2A, Fig. 4E) revealed diffuse and intense cytoplasmic immunoreactivity for both MUC2 (Fig. 2C, Fig. 4F) and MUC5AC (Fig. 2E). The MUC2 immunoreactivity was limited to the cytoplasm of the tumor cells; the adjacent pools of extracellular mucin were nonimmunoreactive. The MUC5AC immunoreactivity was also present mainly in the cytoplasm of the tumor cells but occasionally was also observed in the adjacent extracellular mucin. All of the 15 solitary mucinous tumors of the appendix expressed MUC2 and MUC5AC by in situ hybridization studies with MUC2 signals being greater than MUC5AC signals (Fig. 6B). There was no difference in either the MUC2 or MUC5AC signals with the degrees of malignant transformation (Fig. 6C).

\section{Primary Ovarian Mucinous Tumors}

Of the 75 primary ovarian mucinous tumors chosen for study, 35 were benign, 25 were borderline and 15 were malignant. 2 of the borderline and 3 of the malignant were associated with pseudomyxoma ovarii but not pseudomyxoma peritonei. 12 of the malignant and 3 of the borderline primary ovarian mucinous tumors were, however, associated with peritoneal implants. Were these 3 borderline tumors really borderline? Since our study was a retrospective study designed primarily to examine MUC2 and MUC5AC expression in pseudomyxoma peritonei and related lesions, we were limited to a study of the sections on hand. The question of whether some of the borderline tumors really had foci of microinvasion or intraepithelial carcinoma is a legitimate question that we can not address. The benign primary ovarian mucinous tumors (Fig. 2B) were composed uniformly of 1-2 layers of banal endocervical type lining cells. In 15 of the 35 benign tumors rare intestinal type goblet cells were present. The borderline tumors were characterized by at least five layers of lining cells and an absence of invasion and or severe cytologic atypia. The 15 malignant ovarian mucinous tumors (Fig. 3B) were associated with easily identifiable intestinal type goblet cells. The vast majority of primary mucinous tumors of the ovary did not express MUC2. Every primary ovarian mucinous tumor, on the other hand, showed diffuse cytoplasmic immunoreactivity for MUC5AC (Fig. 2F, Fig. 3F). The MUC5AC immunoreactivity was mainly within the cytoplasm of the tumor cells but occasionally extracellular mucin exhibited focal MUC5AC immunoreactivity. MUC2 immunoreactivity in primary ovarian mucinous tumors was near absent (Fig. 2D, Fig. 3D). In the benign ovarian tumors, MUC2 immunoreactivity was completely absent in 32 of 35 cases; in the borderline tumors, completely absent in 21 of 25 cases; and in the malignant cases, completely absent in 10 of 15 cases. The cases within the benign, borderline and malignant categories which showed some MUC2 immunoreactivity exhibited weak staining (limited to focal staining of less than $5 \%$ of the tumor cells). In none of the cases was their strong diffuse staining. In the 12 cases which showed focal staining, the immunoreactivity was limited to the scattered goblet cells which were present. If we had designated this category of focal staining as negative rather than positive our overall results (Table 2) would be even more dramatic in that none of the primary ovarian mucinous tumors would be positive for MUC2. In nearly all cases, by in situ hybridization, MUC2 signals were also completely absent or were confined to the rare goblet cells which were present in a few cases. 72 of the 75 primary ovarian mucinous tumors expressed MUC5AC by in situ hybridization studies. There was also no significant difference in expression of MUC5AC by either the endocervical or intestinal type cells which were 

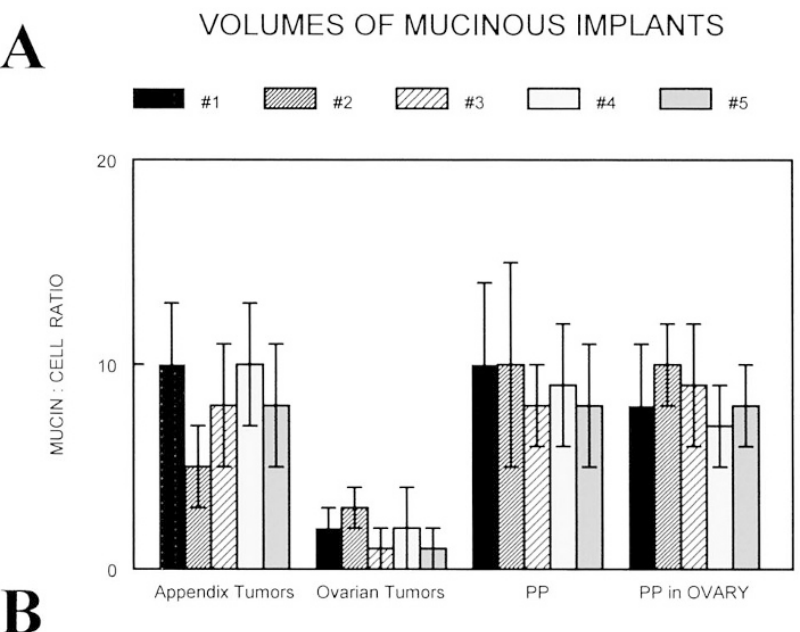

MUC2 / MUC5AC IN SITU HYBRIDIZATION

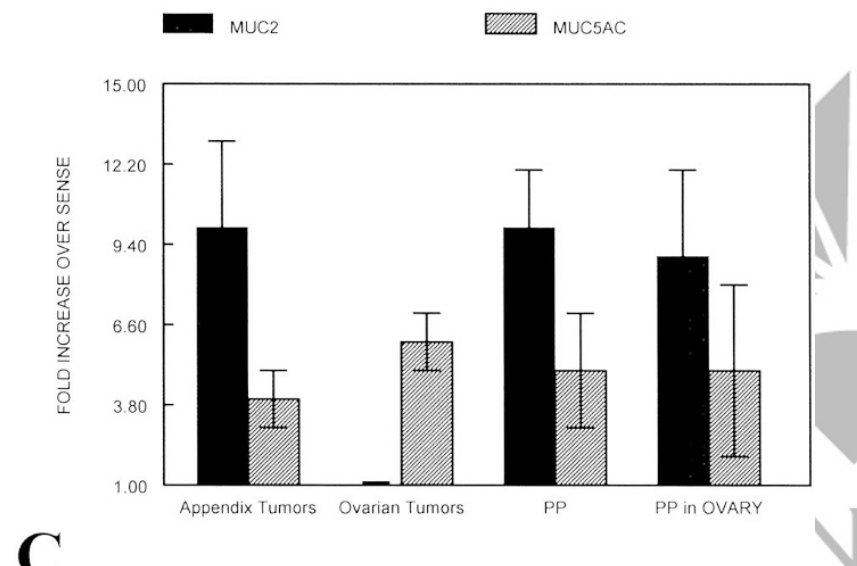

MUC2 / MUC5AC IN SITU HYBRIDIZATION

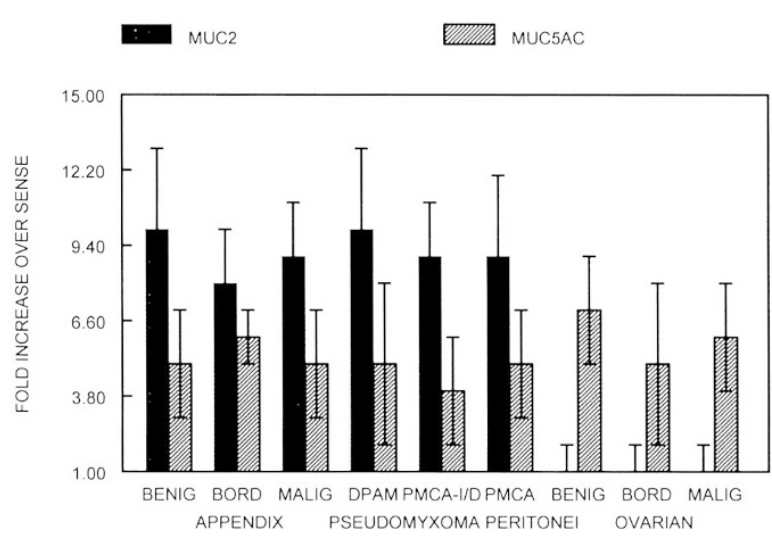

FIGURE 6. (A) The extracellular mucin:cell ratio is depicted in 5 individual cases of mucinous tumors of the appendix, primary ovarian mucinous tumors, generalized pseudomyxoma peritonei and pseudomyxoma peritonei involving the ovary. In each, results depict mean \pm standard deviation of 10 medium power microscopic fields (x250). Higher mucin:cell ratios averaging 10:1 are in evidence in nonovarian tumors presumably due to the contribution of MUC2. (B) The MUC2 and MUC5AC antisense in situ hybridization signals are depicted in various mucinous lesions. Here mean signals from all the individual cases were pooled and an overall mean \pm standard deviation was calculated. Non-ovarian tumors (all within the family of pseudomyxoma peritonei [PP]) exhibit strong signals with MUC2 more than MUC5AC. Ovarian tumors, in contrast, exhibit no MUC2 but slightly higher MUC5AC. (C) The MUC2 and MUC5AC antisense in situ hybridization signals are depicted in the various mucinous lesions showing different degrees of malignant transformation. The pseudomyxoma peritonei cases are divided into disseminated present. There was no difference in either the MUC2 or MUC5AC signals with the degrees of malignant transformation (Fig. 6C). In the cases of primary ovarian mucinous tumors associated with peritoneal implants or pseudomyxoma ovarii, there were no differences in MUC2 or MUC5AC expression between the different sites by either immunocytochemistry or in situ hybridization studies.

\section{Normal Control Tissues}

Sections of normal appendix (Fig. 4A) revealed MUC2 (Fig. 4B) and MUC5AC immunoreactivity confined to the goblet cells. MUC2 and MUC5AC signals were also present within these same cells by in situ hybridization studies (Fig. 5C-D). The nongoblet cells of the appendix were completely negative for MUC2 and MUC5AC by immunocytochemistry and in situ hybridization studies. The surface epithelial (mesothelial) cells of the normal ovary and mesothelial cells of the peritoneum were also completely negative for MUC2 but weakly positive for MUC5AC by both immunocytochemistry and in situ hybridization studies (data not shown).

\section{DISCUSSION}

Our studies have demonstrated that MUC2 is a reliable molecular marker for pseudomyxoma peritonei which epitomizes the central abnormality of the disease, a disease of MUC2 overexpression. MUC2 therefore represents a potential therapeutic molecular target because if MUC2 expression can be inhibited in pseudomyxoma peritonei by pharmacological means, the disease could be better treated. It has been recently demonstrated that "traditional pseudomyxoma peritonei" includes three pathologically, surgically, and prognostically distinctive types of tumors with varying degrees of malignant transformation. These include disseminated peritoneal adenomucinosis, peritoneal mucinous carcinomatosis and peritoneal mucinous carcinomatosis with intermediate or discordant features $(15,20)$ so the degree of transformation of the mucinous epithelium is important in determining the behavior of the disease process. Mucin accumulation is the overwhelming problem in disseminated peritoneal adenomucinosis whereas widespread dissemination and infiltration of carcinoma rather than just mucin accumulation is the problem in peritoneal mucinous carcinomatosis.

\footnotetext{
peritoneal adenomucinosis (DPAM), peritoneal mucinous carcinomatosis with intermediate or discordant features (PMCA-I/D) and peritoneal mucinous carcinomatosis (PMCA). Here mean signals from all the individual cases were pooled and an overall mean \pm standard deviation was calculated. The signals vary according to cell of origin (intestinal $v$ ovarian) but do not vary with the degrees of malignant transformation.
} 
Pseudomyxoma peritonei is sometimes associated with ovarian involvement but the frequency of ovarian involvement has been overstated (14-16). Because ovarian involvement may mimic ovarian carcinoma, for years the disease was thought to take origin from the ovary (6-10). Our findings would confirm, however, the more recent beliefs about the disease that pseudomyxoma peritonei mainly takes origin from mucinous tumors of the appendix and that its involvement with the ovary is due to metastatic spread and not primary origin. Recent studies observing patterns of cytokeratin immunoreactivity $(11,12)$ and loss of heterozygosity (13) have led to this conclusion. Our findings with respect to MUC2 also lead to this conclusion as MUC2 is a gastrointestinal marker and not an ovarian marker (25).

It is sometimes difficult to distinguish a primary ovarian mucinous tumor from metastatic involvement of the ovary by pseudomyxoma peritonei. However when a diagnosis of pseudomyxoma peritonei is obvious, there is no diagnostic problem because the ovarian tumors are metastatic from the appendix in most or probably all cases. It has been presumed that most if not all cases of pseudomyxoma peritonei arise from obvious or occult appendiceal primaries. The problem with the occult appendiceal primary is that often the appendix is "lost to medical followup", either not removed or removed and inadequately sâmpled. In those cases, the conclusion that we are dealing with an occult appendiceal primary requires a presumption. The findings of MUC2 by in situ hybridization and immunocytochemistry in those-cases provide support for an appendiceal primary without requiring any presumption.

Cytokeratin staining has also been used to determine site of origin in cases of pseudomyxoma peritonei whose origin is equivocal. We performed cytokeratin $7 / 20$ immunocytochemistry in our 10 anatomically equivocal pseudomyxoma peritonei cases. 9 of 10 were positive for cytokeratin 20; 8 of 10 were negative for cytokeratin 7 . All 10 were positive for MUC2 as well as MUC5AC by both immunocytochemistry as well as in situ hybridization. Both sets of studies suggested therefore that these 10 anatomically equivocal pseudomyxoma peritonei cases were of gastrointestinal origin. MUC2 immunocytochemistry and in situ hybridization may therefore be useful in the setting where a diagnosis of pseudomyxoma peritonei is suspected but the cytokeratin 20 stain is negative or the cytokeratin 7 stain is positive. If the pattern of cytokeratin staining is $7-/ 20+$, probably MUC2 immunocytochemistry and in situ hybridization would not "add value" from a diagnostic standpoint. From a routine standpoint, cytokeratin stains are much easier to do and interpret than mucin immunocytochemistry and in situ hybridization and certainly that has to be taken into account in the routine workup of these cases. The main value of MUC2 over cytokeratin presently is more theoretical than practical: cytokeratin gene expression of pseudomyxoma peritonei has nothing to do with its morbidity or mortality whereas MUC2 has a lot to do with it especially with the disseminated peritoneal adenomucinosis variant.

Primary mucinous tumors of the appendix including adenomas, borderline or uncertain malignant potential and adenocarcinomas (5) all can give rise to pseudomyxoma peritonei. Alternately the appendix, being an organ prone to obstruction, may spontaneously rupture disseminating implants of epithelial cells and producing pseudomyxoma peritonei $(7,8)$. Interestingly some of the early studies in the 1950's produced pseudomyxoma peritonei experimentally by ligating the appendix in rabbits (29).

It is interesting that in the early studies out of Johns Hopkins and Mayo Clinic (30), more women than men were initially observed to have pseudomyxoma peritonei. In a study of pseudomyxoma peritonei from Memorial SloanKettering (31), more women than men were also observed to have pseudomyxoma peritonei. Perhaps in some of these earlier studies primary ovarian neoplasms were erroneously included in the overall numbers falsely giving rise to a female predominance. In more recent studies consisting of comparatively larger number of patients, a male predominance (male:female ratio of 60:40) was observed (15).

Since the common element in pseudomyxoma peritonei especially of the disseminated peritoneal adenomucinosis category was mucin accumulation, it made sense to us to study the mucin which was secreted in light of the recent cloning of a number of human mucin genes $(21,22)$. We chose to specifically study MUC2 and MUC5, because they were known to be secretory mucins which had the physicochemical property of gel forming (23, 24 ), a property common to the gelatinous nature of pseudomyxoma peritonei. Our study also focused on MUC2 and MUC5AC because recent studies had observed differential expression of these 2 mucins in gastrointestinal $v$ ovarian neoplasms (25). MUC2 had been known to be expressed in both the gastrointestinal tract as well as the bronchus (32-35). MUC5AC had also been observed to be expressed in both of these sites as well as the endocervix and endometrium (36). Occasionally aberrant expressions of these mucins had been observed in cancers arising from organs where the particular mucins are not normally expressed. Recently we observed, for example, that both MUC2 and MUC5AC are expressed in colloid carcinomas of the breast, an or- 
gan in which MUC2 and MUC5AC are not normally expressed (24).

Our studies indicate that based on the mucin expression profile that mucinous tumors of the appendix, primary ovarian mucinous tumors and pseudomyxoma peritonei all express MUC5AC but only mucinous tumors of the appendix and their associated pseudomyxoma peritonei express MUC2. This pattern of mucin expression reflects their alleged cells of origin. Goblet cells of the appendix express both MUC2 and MUC5AC. This suggests that they are the cell of origin of mucinous tumors of the appendix and pseudomyxoma peritonei. Mesothelial cells and cells which line the surface of the ovary express only MUC5AC. This suggests that they are the cell of origin of mucinous tumors of the ovary. Specifically this pattern of mucin expression suggests that pseudomyxoma peritonei does not originate from either the surface epithelium of the ovary or the mesothelium of the peritoneum.

MUC2 expression was observed in our study in a minority of primary ovarian mucinous tumors. 3 of 35 benign ovarian mucinous tumors, 4 of 25 borderline ovarian mucinous tumors and 5 of 15 malignant ovarian mucinous tumors exhibited weak MUC2 immunoreactivity (limited to focal staining of less than $5 \%$ of the tumor cells). In these cases, the immunoreactivity localized to the scattered goblet cells which were present. If we had designated this category of focal staining as negative rather than positive our overall results (Table 2) would be even more dramatic in that none of the primary ovarian mucinous tumors would be positive for MUC2. Dong et al. (37) and Tashiro et al. (38). using immunocytochemistry reported MUC2 expression in a low percentage of borderline and a higher percentage of malignant ovarian mucinous tumors. Dong et al. reported that as many as 55 percent of malignant ovarian mucinous tumors were immunoreactive for MUC2. Their pattern of staining was characterized as cytoplasmic in character, focal in distribution and predominantly limited to the intestinal goblet cells. Their pattern and distribution of staining is similar to our observations. The discrepancy in the number of positive cases between their studies and our studies may be due to the smaller number of malignant ovarian mucinous tumors in our study (since we were primarily interested in studying pseudomyxoma peritonei and not ovarian carcinoma). We believe, however, that this focal MUC2 immunoreactivity does not account for the extracellular mucin deposits observed in primary ovarian mucinous tumors, their associated peritoneal implants or in the cases of pseudomyxoma ovarii where MUC5AC is by far the dominant mucin expressed in all of the cells. The finding of MUC2 in malignant ovarian tumors in these other studies could also be due to misclassification of gastrointestinal carcinoma metastases as primary ovarian mucinous carcinomas. Hanski et al. (39). reported that MUC2 was expressed at the protein and mRNA levels in virtually every malignant primary ovarian mucinous tumor using immunocytochemistry and RT-PCR. Our conflicting results with this study raise several possibilities other than the smaller number of cases in our study. Because pseudomyxoma peritonei can involve the ovaries, some of the cases in the Hanski study might be cases of pseudomyxoma peritonei. Another possibility is that the specificities of the different monoclonal MUC2 antibodies used in the two studies may be different. The monoclonal antibody used in the Hanski study reportedly reacts with both gastric and tracheobronchial mucin (40) and therefore may be cross-reacting with MUC5AC. The monoclonal antibody we used in our study specifically did not cross react with MUC5AC. The demonstration by RT-PCR of MUC2 transcripts in borderline and malignant primary ovarian mucinous tumors has been confirmed by another study which observed MUC2 not only in primary ovarian mucinous tumors but also in many other benign and malignant surface epithelial ovarian tumors as well as normal ovarian tissue (41). Illegitimate transcription of many genes occurs in many tissues and can be demonstrated with sensitive enough assays such as RT-PCR. The fact that we, using in situ hybridization, have demonstrated that MUC5AC and not MUC2 is detected in primary ovarian mucinous tumors proves that MUC5AC is the dominant mucin expressed in these tumors, irrespective of the low levels of MUC2 which might also be present.

Finally not only does MUC2 serve as a marker of pseudomyxoma peritonei but likely accounts for the differences in the gross appearances of the mucinous deposits of pseudomyxoma peritonei compared to the mucinous implants of ovarian carcinoma. Our findings revealed that the mucin:cell ratio averaged 10 to 1 in all of the cases of pseudomyxoma peritonei whether there was an associated mucinous tumor of the appendix or ovarian involvement. We have even observed some cases of pseudomyxoma peritonei where the mucin:cell is as much as 100:1 or even 1000:1. Sometimes the mucin accumulations are so abundant that intestinal cells can not even be identified. We did not include those cases in this report because our present study requires the presence of significant numbers of intestinal lining cells for immunocytochemistry and in situ hybridization. Primary ovarian neoplasms, in contrast, gave rise to peritoneal implants where the mucin:cell ratio was only 1 to 1 . Since both pseudomyxoma peritonei and ovarian neoplasms express MUC5AC, it likely is the 
MUC2 which is responsible for the high degree of gelation within the pseudomyxoma peritonei deposits. The complete lack of anti-MUC2 to detect the core protein within the extracellular mucin deposits of pseudomyxoma peritonei compared to the limited ability of anti-MUC5AC to reach its core protein is indirect evidence that extracellular MUC2 is more extensively glycosylated than MUC5AC and therefore sterically occupies a greater volume than MUC5AC on an equimolar basis. It would then be anticipated that extracellular mucin deposits of MUC2 would occupy a greater volume than the extracellular mucin deposits of MUC5AC and cause pseudomyxoma peritonei to have its gross "jelly belly" appearance.

It is again important to realize that not all of what has been classified as pseudomyxoma peritonei historically exhibits the same biology or the same degree of mucin accumulation. The historical emphasis on the presence and amount of mucin without regard to the degree of malignant transformation of the mucinous epithelium was one of the main reasons for the confusion in the older literature about the nature and behavior of all things classified as pseudomyxoma peritonei without regard to the nature of the tumor responsible for the condition $(15,20)$. It must be realized that it is the nature of the epithelium that drives the prognosis even though most cases have abundant mucin production. In particular it is not even the cellularity in and of itself that is prognostic - what matters is the degree of malignancy of the epithelium. Malignant cases tend to be more cellular than the benign ones and there are distinctive surgical findings in each. Experienced surgeons can recognize these differences because disseminated peritoneal adenomucinosis has the characteristic "jelly belly" appearance whereas peritoneal mucinous carcinomatosis has the appearance of disseminated metastatic carcinoma even when it produces abundant mucin. This established clinical difference is important to note in light of our data which showed equal expression of the gel-forming MUC2 independent of the degree of malignant transformation present. If both benign and malignant appendiceal tumors produce the same mucin, why is disseminated peritoneal adenomucinosis (the true pseudomyxoma peritonei derived from a ruptured appendiceal adenoma) clinically and surgically distinct from peritoneal mucinous carcinomatosis (a process that does not really produce the classic "jelly belly" and is derived from appendiceal mucinous carcinoma)? It must be appreciated that many factors in addition to the rates of mucin expression may be responsible for the accumulation of extracellular mucin including the rates of mucin secretion, on one hand, and the time that the mucin has to accumulate and the anatomical locations where it accumu- lates on the other hand. One could envision that a benign neoplasm such as disseminated peritoneal adenomucinosis which secretes mucin but which is also slow growing, less invasive with more of a penchant for producing superficial implants on surfaces and cavities and which does not kill the patient except after many years would produce massive mucin accumulations as the "jelly belly". On the other hand a malignant neoplasm such as peritoneal mucinous carcinomatosis which secretes mucin but whose cells are rapidly growing, more invasive with a penchant for deep parenchymal invasion of internal viscera and which kills the patient rapidly, would not accumulate as much mucin.

Still MUC2 is the primary molecular marker for all types of pseudomyxoma peritonei. Our recent molecular studies with pseudomyxoma peritonei and derived primary cell cultures have indicated that MUC2 expression is epigenetically regulated and susceptible to pharmacological regulation (42). This suggests that MUC2 may also be the primary molecular target for pseudomyxoma peritonei as well.

Acknowledgments: The authors wish to thank Ehud Drori and Carol B. Basbaum of the Department of Anatomy, UCSF School of Medicine, San Francisco, CA for assistance and advice with the in situ hybridization studies. This study was supported by a seed grant from the Jonsson Comprehensive Cancer Center, UCLA School of Medicine, Los Angeles, CA 90024.

\section{REFERENCES}

1. Weaver C. Mucocele of the appendix with pseudomucinous degeneration. Am J Surg 1937;36:523-6.

2. Young RH, Gilks CB, Scully RE. Mucinous tumors of the appendix associated with mucinous tumors of the ovary and pseudomyxoma peritonei. A clinicopathological analysis of 22 cases supporting an origin in the appendix. Am J Surg Pathol 1991;15:415-29.

3. Carleton CC. Mucoceles of the appendix and peritoneal pseudomyxoma. Arch Pathol 1955;60:39-48.

4. Parsons J, Gray GF, Thorbjarnarson B. Pseudomyxoma peritonei. Arch Surg 1970;101:545-9.

5. Carr NJ, Sobin LH. Unusual tumors of the appendix and pseudomyxoma peritonei. Semin Diagn Pathol 1996;13:31425 .

6. Klemi PJ. Pathology of mucinous ovarian cystadenomas. I. Argyrophil and argentaffin cells and epithelial mucosubstances. Acta Pathol Microbiol Scand [A] 1978;86A:465-70.

7. Gough DB, Donohue JH, Schutt AJ, et al. Pseudomyxoma peritonei. Long-term patient survival with an aggressive regional approach. Annu Surg 1994;219:112-9.

8. Costa MJ. Pseudomyxoma peritonei. Histologic predictors of patient survival. Arch Pathol Lab Med 1994;118:1215-9.

9. Seidman JD, Elsayed AM, Sobin LH, Tavassoli FA. Association of mucinous tumors of the ovary and appendix. A clinicopathologic study of 25 cases. Am J Surg Pathol 1993;17: 22-34. 
10. Sumithran E, Susil BJ. Concomitant mucinous tumors of appendix and ovary. Result of a neoplastic field change? Cancer 1992;70:2980-3.

11. Guerrieri C, Franlund B, Fristedt S, Gillooley JF, Boeryd B. Mucinous tumors of the vermiform appendix and ovary, and pseudomyxoma peritonei: histogenetic implications of cytokeratin 7 expression. Hum Pathol 1997;28:1039-45.

12. Ronnett BM, Shmookler BM, Diener-West M, Sugarbaker $\mathrm{PH}$, Kurman RJ. Immunohistochemical evidence supporting the appendiceal origin of pseudomyxoma peritonei in women. Int J Gynecol Pathol 1997;16:1-9.

13. Szych C, Staebler A, Connolly DC, et al. Molecular genetic evidence supporting the clonality and appendiceal origin of Pseudomyxoma peritonei in women. Am J Pathol 1999;154: 1849-55.

14. Sugarbaker PH, Ronnett BM, Archer A, et al. Pseudomyxoma peritonei syndrome. Adv Surg 1996;30:233-80.

15. Ronnett BM, Zahn CM, Kurman RJ, Kass ME, Sugarbaker PH, Shmookler BM. Disseminated peritoneal adenomucinosis and peritoneal mucinous carcinomatosis. Am J Surg Pathol 1995; 19:1390-408.

16. Ronnett BM, Kurman RJ, Zahn CM, Shmookler BM, Jablonski KA, Kass ME, et al. Pseudomyxoma peritonei in women. Hum Pathol 1995;26:509-524.

17. Jackson SL, Fleming RA, Loggie BW Geisinger KR. Gelatinous ascites: a cytohistologic study of pseudomyxoma peritonei in 67 patients. Mod Pathol 2001;14:664-71.

18. Sugarbaker PH, Ronnett BM, Archer A, et al. Pseudomyxoma peritonei syndrome. Adv Surg 1996;30:233-80.

19. Esquivel J, Sugarbaker PH. Clinical presentation of the Pseudomyxoma peritonei syndrome. $\mathrm{Br}$ J Surg 2000;87:1414-8.

20. Ronnett BM, Yan $\mathrm{H}$, Kurman RJ, et al. Patients with pseudomyxoma peritonei associated with disseminated peritoneal adenomucinosis have a significantly more favorable prognosis than patients with peritoneal mucinous carcinomatosis. Cancer 2001;92:85-91.

21. Gendler SJ, Spicer AP. Epithelial mucin genes. Annu Rev Physiol 1995;57:607-34.

22. Verma M, Davidson EA. Mucin genes: structure, expression and regulation. Glycoconj J 1994;11:172-9.

23. Kim YS, Gum JR Jr. Diversity of mucin genes, structure, function, and expression. Gastroenterology 1995;109:9991001.

24. O'Connell JT, Shao ZM, Drori E, Basbaum CB, Barsky SH. Altered mucin expression is a field change that accompanies mucinous (colloid) breast carcinoma histogenesis. Hum Pathol 1998;29:1517-23.

25. Albarracin CT, Jafri J, Montag AG, Hart J, Kuan SF. Differen tial expression of MUC2 and MUC5AC mucin genes in primary ovarian and metastatic colonic carcinoma. Hum Pathol 2000;31:672-7.

26. Taylor CR, Shi SR, Chaiwun B, et al. Strategies for improving the immunohistochemical staining of various intranuclear prognostic markers in formalin-paraffin sections: androgen receptor, estrogen receptor, progesterone receptor, p53 pro- tein, proliferating cell nuclear antigen, and Ki-67 antigen revealed by antigen retrieval techniques. Hum Pathol 1994; 25:263-70.

27. Li JD, Dohrman AF, Gallup M, et al. Transcriptional activation of mucin by Pseudomonas aeruginosa lipopolysaccharide in the pathogenesis of cystic fibrosis lung disease. Proc Natl Acad Sci USA 1997;94:967-72.

28. Chang SK, Dohrman AF, Basbaum CB, et al. Localization of mucin (MUC2 and MUC3) messenger RNA and peptide expression in human normal intestine and colon cancer. Gastroenterology 1994;107:28-36.

29. Cheng KK. An experimental study of mucocele of the appendix and pseudomyxoma peritonei. J Path Bact 1954;61:21725.

30. Woodruff R, McDonald JR. Benign and malignant cystic tumors of the appendix. Surg Gynecol Obst 1938;61:750-5.

31. Smith JW, Kemeny N, Caldwell C, Banner P, Sigurdson E, Huvos A. Pseudomyxoma peritonei of appendiceal origin. Cancer 1992;70:396-401.

32. Devine PL, Birrell GW, Whitehead RH, et al. Expression of MUC1 and MUC2 mucins by human tumor cell lines. Tumour Biol 1992;13:268-77.

33. Allen A, Hutton DA, Pearson JP. The MUC2 gene product: a human intestinal mucin. Int J Biochem Cell Biol 1998;30: 797-801.

34. Blank M, Klussmann E, Kruger-Krasagakes S, et al. Expression of MUC2-mucin in colorectal adenomas and carcinomas of different histological types. Int J Cancer 1994;59: 301-6.

35. Xing PX, Prenzoska J, Layton GT, Devine PL, McKenzie IF. Second-generation monoclonal antibodies to intestinal MUC2 peptide reactive with colon cancer. J Natl Cancer Inst 1992;84:699-703.

36. Gipson IK, Ho SB, Spurr-Michaud SJ, et al. Mucin genes expressed by human female reproductive tract epithelia. Biol Reprod 1997;56:999-1011.

37. Dong Y, Walsh MD, Cummings MC, et al. Expression of MUC1 and MUC2 mucins in epithelial ovarian tumours. J Pathol 1997;183:311-7.

38. Tashiro Y, Yonezawa S, Kim YS, Sato E. Immunohistochemical study of mucin carbohydrates and core proteins in human ovarian tumors. Hum Pathol 1994;25:364-72.

39. Hanski C, Hofmeier M, Schmitt-Graff A, et al. Overexpression or ectopic expression of MUC2 is the common property of mucinous carcinomas of the colon, pancreas, breast, and ovary. J Pathol 1997;182:385-91.

40. Devine PL, McGuckin MA, Birrell GW, et al. Monoclonal antibodies reacting with the MUC2 mucin core protein. Br J Cancer 1993;67:1182-8.

41. Giuntoli RL 2nd, Rodriguez GC, Whitaker RS, Dodge R, Voynow JA. Mucin gene expression in ovarian cancers. Cancer Res 1998;58:5546-50.

42. O'Connell JT, Tomlinson JS, Roberts AA, McGonigle K, Barsky SH. Pseudomyxoma peritonei is a disease of MUC2expressing goblet cells. Am J Pathol 2002; 161:551-64. 